\title{
Properties of dayside nonlinear rising tone chorus emissions at large $L$ observed by GEOTAIL
}

\author{
Olga P. Verkhoglyadova ${ }^{1,2}$, Bruce T. Tsurutani ${ }^{3}$, Y. Omura ${ }^{2}$, and S. Yagitani ${ }^{4}$ \\ ${ }^{1}$ University of California, Riverside, USA \\ ${ }^{2}$ RISH, Kyoto University, Uji, Kyoto, Japan \\ ${ }^{3}$ Jet Propulsion Laboratory, CalTech, Pasadena, USA \\ ${ }^{4}$ Kanazawa University, Kanazawa, Japan
}

(Received September 10, 2007; Revised November 11, 2007; Accepted December 20, 2007; Online published May 29, 2009)

\begin{abstract}
This paper studies some wave properties of nonlinear rising tone chorus emissions in the outer regions of the dayside equatorial magnetosphere at $\sim\left(6.3,-4.7,0.7 R_{\mathrm{E}}\right)$ in GSE coordinates. We analyze data obtained with the PWI and WFC receivers on GEOTAIL associated with a substorm on April 29, 1993. Fine structure of the chorus elements and inter-element spacings are shown. Directions of propagation of the chorus elements relative to the local magnetic field lines are analyzed. Wave polarizations, intensities and spectral properties of chorus in the equatorial Earth's magnetosphere are discussed.
\end{abstract}

Key words: Chorus emissions, wave-particle interactions, electron acceleration.

\section{Introduction}

Major chorus statistical properties have been presented in Tsurutani and Smith (1974, 1977), Nagano et al. (1996) and Meredith et al. (2001, 2003). Recently, progress has been made in studies of wave properties in the generation region, such as fine structure and propagation of chorus (Santolik et al., 2003, 2004) using multipoint Cluster measurements. We will summarize some relevant chorus characteristics below.

Chorus ELF waves are observed in the outer magnetosphere at $L>5 R_{\mathrm{E}}$ primarily in two latitude regions, and have been classified into equatorial and high-latitude chorus (Tsurutani and Smith, 1977). Equatorial chorus $\left(\mathrm{MLAT}<15^{\circ}\right)$ occurs during substorms with the peak intensity near the magnetic equator. These waves are believed to be generated by trapped substorm electrons via a cyclotron resonance instability (see also Lauben et al., 2002). Highlatitude chorus is detected near the magnetopause and has little apparent connection with substorm activity. Our study is focused on the near-equatorial dayside chorus waves.

Equatorial chorus is an electromagnetic whistler mode wave observed as two distinct bands, one above and one below 0.5 of the local electron gyrofrequency, $v_{\text {ce }}$ (Tsurutani and Smith, 1974). The wave absence at $0.5 v_{\text {ce }}$ can be explained by Landau damping. Chorus is often composed of $\sim 0.5$ sec elements. There are several types of frequencytime chorus structures: rising and falling tones, and narrowband (structureless) chorus. Chorus is a nonlinear emission. Its elements are composed of sub-elements or wave packets separated by intervals of noise (Santolik et al., 2003). Chorus plays an important role in wave-particle interac-

Copyright (c) The Society of Geomagnetism and Earth, Planetary and Space Sciences (SGEPSS); The Seismological Society of Japan; The Volcanological Society of Japan; The Geodetic Society of Japan; The Japanese Society for Planetary Sciences; TERRAPUB tions in the outer magnetosphere (Tsurutani and Lakhina, 1997; Horne et al., 2005). An up-to-date review of the chorus-driven electron acceleration based on CRRES observations was made by Meredith et al. (2001, 2003). Computer modeling results on relativistic electron interaction with whistler mode waves were reported by Omura and Summers (2004). In the case of a chorus element, contribution to the pitch-angle diffusion from a nearly monochromatic large-amplitude sub-element(s) can be much larger than from an average wave field of the whole element. The main focus of this study is on properties of nonlinear chorus emissions which could be important for wave-particle interactions, i.e., fine structure of the chorus waves, direction of propagation, polarization and magnitudes of the magnetic field disturbances.

\section{Analysis of the GEOTAIL Data}

We present first results on four time intervals of wave activity during a substorm interval starting at $\sim 2307$ UT of April 29, 1993. A major negative excursion of the AL index started at $\sim 2100$ UT. Assuming chorus wave generation by gradient-drifting of 10-40 keV electrons, a delay time between the onset of chorus and a substorm is estimated as 2 hrs (Tsurutani and Smith, 1974). Equatorial daytime chorus is expected to have maximum intensity at $\sim 1000$ LT due to the dayside maximum of energetic electron precipitation. This timing agrees with the beginning of rising tone chorus observations by GEOTAIL around $~ 930$ LT. The satellite was located in the near-equatorial magnetosphere at $\sim(6.3$, $-4.7,0.7 R_{\mathrm{E}}$ ) (in GSE coordinates) and the geocentric distance of $\simeq 7.9 R_{\mathrm{E}}$ at the time of these observations. Intense chorus emissions were observed with the PWI and WFC instruments (Matsumoto et al., 1993; Nagano et al., 1996) onboard GEOTAIL. The PWI (plasma wave instrument) consists of a 3-component search-coil and 2 wire (elec- 
Table 1. Properties of rising tone elements (see text for details).

\begin{tabular}{|c|c|c|c|c|c|c|c|c|c|}
\hline Start time & $\Delta t, \mathrm{sec}$ & $v_{1,2}, \mathrm{kHz}$ & $\Delta v, \mathrm{~Hz}$ & $v_{0}, \mathrm{kHz}$ & $v_{\mathrm{ce}}, \mathrm{kHz}$ & $B_{\max }, \mathrm{pT}$ & $\theta,^{\circ}$ & $\theta_{\mathrm{G}}, \stackrel{\circ}{ }$ & $\theta_{\mathrm{r}}, \stackrel{\circ}{ }$ \\
\hline $2306: 55$ & 0.3 & $0.9, \quad 0.5$ & 220 & 0.8 & 3.44 & 80 & 11 & 62 & 77 \\
\hline $2306: 55$ & 0.15 & $0.75,0.62$ & 130 & 0.7 & 3.44 & 70 & 29 & 66 & 78 \\
\hline $2311: 39$ & 0.3 & $0.95,0.55$ & 400 & 0.75 & 3.51 & 200 & 17 & 65 & 78 \\
\hline 2321:07 & 0.68 & $1.1, \quad 0.65$ & 450 & 0.875 & 3.50 & 300 & 42 & 60 & 76 \\
\hline 2349:31 & 0.7 & $1.1, \quad 0.65$ & 450 & 0.875 & 3.03 & 100 & 22 & 55 & 73 \\
\hline $2349: 31$ & 0.6 & 0.6 & 400 & 0.8 & 3.03 & 100 & 22 & 58 & 75 \\
\hline
\end{tabular}
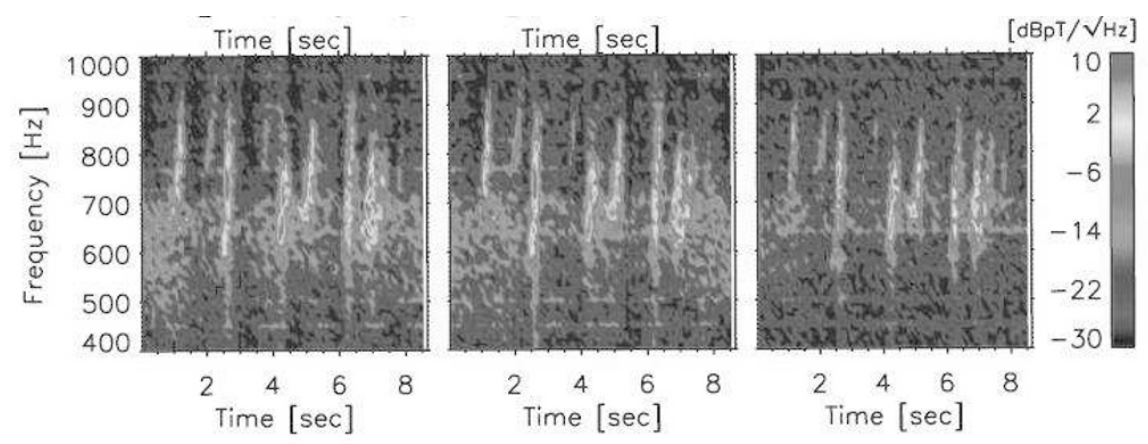

Fig. 1. Time-frequency power spectra of three magnetic field components in GSM coordinates derived from WFC measurements onboard GEOTAIL. The beginning of the interval corresponds to 2306:55 UT of April 29, 1993. The left, central and right panels correspond to $B_{x}, B_{y}$ and $B_{z}$ components.

tric) antennas with sensitivity of $1.5 \times 10^{-5} \mathrm{nT} / \mathrm{Hz}^{1 / 2}$ for the magnetic field and $8.0 \times 10^{-9} \mathrm{~V} /\left(\mathrm{m} \mathrm{Hz}^{1 / 2}\right)$ for the electric field, respectively. The WFC (wave form capture) receiver samples $8.7 \mathrm{sec}$ snapshots every $5 \mathrm{~min}$ between $10 \mathrm{~Hz}$ and $4 \mathrm{kHz}$. For each of the four time intervals of $\sim 8.7 \mathrm{sec}$ duration we select several distinct rising tone chorus elements. Properties of 6 such elements are summarized in Table 1. The following parameters were estimated: $\Delta t$ is the duration of a rising tone element, $v_{1,2}$ are the maximum and minimum frequencies within the element, $\Delta v$ is the frequency band (bandwidth at half-maximum), $v_{0}$ - average frequency of the tone, $B_{\max }$ - the maximum magnitude of magnetic field disturbance in the chorus, $\theta$ - the propagation angle to the local magnetic field line, $\theta_{\mathrm{G}}$ - the local Gendrin angle calculated for $v_{0}$, and $\theta_{\mathrm{r}}$ is the resonance angle. Average magnetic field components measured at $\sim 2306: 55$ UT were (26.1, 52.1, 108.2 nT) is GSE coordinate system. An example of chorus is shown in Fig 1.

We process magnetic field waveforms in the principal or the minimum variance analysis (MVA) frame to find direction of propagation of the waves (Tsurutani and Smith, 1977), which corresponds to a direction of minimum variation. As it follows from the chorus generation mechanism, the most intense elements correspond to waves generated locally and propagating under small angles $\theta$ to the local magnetic fieldline (see also Goldstein and Tsurutani, 1984). Whereas waves generated far away have smaller magnitudes (due to Landau damping) and may be propagating obliquely to the local magnetic field line. Our results in Table 1 show that observed intense chorus emissions propagate under relatively small angles to the local magnetic field line. We found that chorus sub-elements sometimes have different directions of propagation relative to the background magnetic field.

The maximum angle of electromagnetic wave propaga-

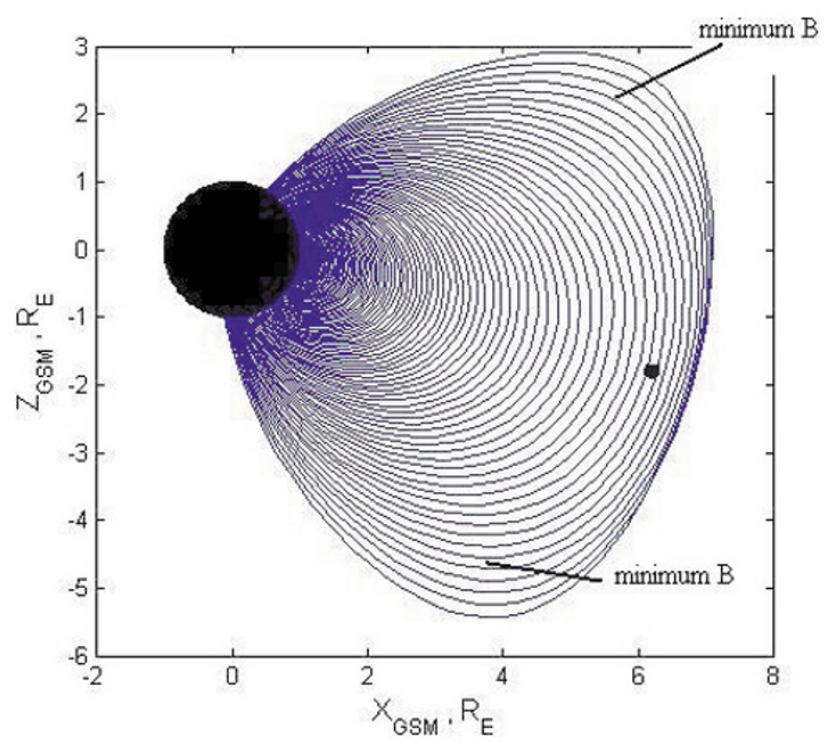

Fig. 2. A schematic view of the inner magnetosphere distorted by the solar wind compression obtained with the Tsyganenko model (Tsyganenko, 2002). A projection into noon local time plane is shown. The projection of the GEOTAIL spacecraft position into the $(X Z)$ plane is indicated by a small dot. "Minimum $B$ pockets" are shown.

tion relative to the magnetic field line is the resonance angle, $\cos \left(\theta_{\mathrm{r}}\right)=v / v_{\mathrm{ce}}($ Goldstein and Tsurutani, 1984), presented in the last column of Table 1. The "Gendrin" angle, shown in the next to the last column of Table 1, is defined in linear plasma theory and corresponds to a minimum value of the refractive index parallel to the magnetic field line and is defined locally as $\cos \left(\theta_{\mathrm{G}}\right)=2 v / v_{\mathrm{ce}}$ (Gendrin, 1961). At the same time, the Gendrin angle is the upper limit for the angle $\theta$. The condition for angular region $\theta= \pm \theta_{\mathrm{G}}$ indicates a proximity to wave generation region for the lower-band 
(a)
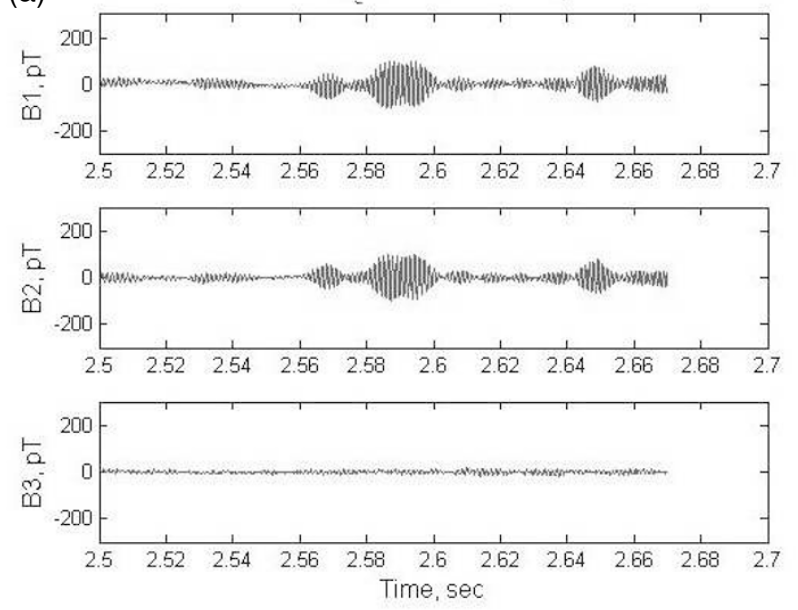

(b)

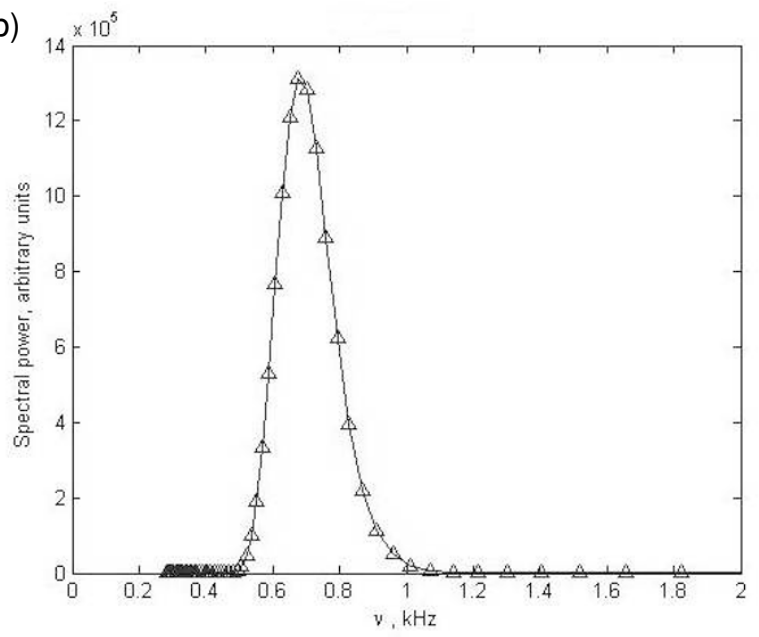

Fig. 3. (a) Example of magnetic field waveform of a chorus element shown in the principal frame. $B_{1}, B_{2}$ and $B_{3}$ denote the maxumum, intermediate and minimum variance directions, respectively. Notice sub-elements separated by intervals of noise. (b) Instantaneous spectrum of the chorus sub-element at $t \simeq 2.585 \mathrm{sec}$ obtained by using Morlet wavelet.

chorus $\left(v_{0}<0.5 v_{\text {ce }}\right)$ (Lauben et al., 2002). The estimated $\theta_{\mathrm{G}}$ in the Table 1 are quite large and are not close to $\pm \theta$.

By the definition and given constant wave frequency, $\theta_{\mathrm{G}}$ is larger in the areas of large background magnetic field, $B_{0}$, and smaller in the "minimum $B$ pockets" (Tsurutani and Smith, 1977). What was the Geotail position in the dipole magnetic field? Figure 2 presents schematic view of the noon local time cross-section of the inner magnetosphere in the GSM coordinates. This sketch was produced with the Tsyganenko model (Tsyganenko, 2002) under undisturbed conditions. The beginning of the interval of Geotail observations was characterized by a very quiet ring current with minumum SYM-H $\simeq-10 \mathrm{nT}$. For this model application we choose Dst $=0$, IMF $B_{z}=0$ and $B_{y}=0$ (in GSM) and the solar wind dynamical pressure equal to $6 \mathrm{nPa}$. The magnetic dipole axis is tilted towards the $X$-axis of the GSM coordinate system.

A distortion (compression) of the outer dayside magnetosphere field lines as compared to a dipole field is clearly shown. Areas of minimum $B_{0}$ are situated at high magnetic latitudes and at large $L\left(r>6 R_{\mathrm{E}}\right)$. This sketch is similar to that in Tsurutani and Smith (1977). The small dot on the right side of the plot is the GEOTAIL position in the $(X Z)$ plane. The GEOTAIL GSM coordinates were $\sim(6.3,-4.4$, $\left.-1.7 R_{\mathrm{E}}\right)$. Thus, the satellite at the time of chorus measurements was located at the magnetic equator and away from the "minimum $B$ pockets".

We use FFT and Morlet wavelet analyses to study spectral properties of the chorus. The wavelet analysis is often applied to study spectral characteristics of a signal with varying time frequencies (Torrence and Compo, 1998). We use the Morlet wavelet with adjustable resolution in time and frequency to calculate instantaneous spectra of chorus elements as well as standard wave spectra in the timefrequency variables. Distinct spectral features within chorus elements corresponding to sub-elements or wave packets were detected. Similar analysis was performed by Santolik et al. (2003, 2004) based on Cluster data. The peak frequency in our study corresponds to about $(0.15-0.4) v_{\text {ce }}$ (see Table 1). Thus, only the lower-band chorus elements $\left(v_{0}<0.5 v_{\text {ce }}\right)$ were analyzed. Figure 3(a) shows an example of one chorus element in the MVA frame consisting of wave packets or sub-elements. One can notice that the wave is circularly polarized. The spectrum of a chorus sub-element is a narrow-band signal (Fig. 3(b)). Bandwidths at halfmaxima for the chorus elements are presented in Table 1. The spectra of wave packets are narrower, and correspond to only a part of the riser element. Wavelet analysis revealed that the wave packet intervals showed small changes in phase, and noise intervals showed large changes in phase. The latter can be explained by random character of disturbances in noise intervals and corresponding rotations of the polarization plane.

We estimate spectral wave intensities for a sub-element and for the whole element presented in the first line of Table 1 . Wave intensity of the wave packet is $\sim 5.710^{-3} \mathrm{nT}^{2}$ compared to $\sim 7.410^{-4} \mathrm{nT}^{2}$ average over the whole element, showing increase by the factor of about 8 . The ratio of the wave packet intensity to intensity of the inter-element spacing $\left(\sim 610^{-5} \mathrm{nT}^{2}\right)$ is thus about two orders of magnitude.

\section{Conclusions}

The study addresses some properties of the rising tone chorus emissions observed with PWI and WFC instruments onboard GEOTAIL during a substorm of April 29, 1993. Most of the chorus waves were found to propagate under small angles $\left(\sim 11^{\circ}-22^{\circ}\right)$ relative to the ambient magnetic field lines. The waves were not propagating at the Gendrin angles. GEOTAIL was located near the magnetic equator and away from the "minimum $B$ pockets" (Tsurutani and Smith, 1977). We use the Tsyganenko model to illustrate that the outer magnetosphere was compressed, even under very quiet conditions. Correct interplanetary data are needed to map the "minimum $B$ pockets" more accurately.

These waves are nearly parallel propagating and are presumably generated close to the GEOTAIL location at large $L$ in the vicinity of the near-equatorial magnetopause. Tim- 
ing of the chorus observation correspond roughly to time delays of substorm electrons drifting from the midnight sector. There is a possibility that magnetospheric compression due to the solar wind ram pressure enhances chorus generation at large $L$-shells (Zhou et al., 2003). Chorus can be intensified by drift-shell splitting. These mechanisms require further studies.

Chorus emissions show distinct fine structure with wave packets separated by intervals of noise (Santolik et al., 2003). We apply FFT and Morlet wavelet transforms to estimate spectral wave intensities for a wave packet or subelement and the whole element. It is shown that the chorus emissions have higher intensities $\left(>10^{-3} \mathrm{nT}^{2}\right)$ inside subelements compared to the average intensity of the whole element by the factor of $\sim 8$.

Acknowledgments. Work of O.V. at RISH, Kyoto University was supported by JSPS Fellowship.

\section{References}

Gendrin, R., Le guidage des whistlers par le champ magnetique, Planet. Space Sci., 5, 274, 1961.

Goldstein, B. E. and B. T. Tsurutani, Wave normal directions of chorus near the equatorial source region, J. Geophys. Res., 89(A5), 2789, 1984.

Horne, R. B., R. M. Thorne, Yu. Y. Sprits et al., Wave acceleration of electrons in the Van Allen radiation belts, Nature, 437(7056), 227, 2005.

Lauben, D. S., U. S. Inan, T. F. Bell, and D. A. Gurnett, Source characteristics of ELF/VLF chorus, J. Geophys. Res., 107(A12), 1429, doi:10. 1029/2000JA003019, 2002.

Matsumoto, H., I. Nagano, R. R. Anderson et al., Plasma wave observations with GEOTAIL spacecraft, J. Geomag. Geoelectr., 46, 59, 1993.

Meredith, N. P., R. B. Horne, and R. R. Anderson, Substorm dependence of chorus amplitudes: implications for the acceleration of electrons to relativistic energies, J. Geophys. Res., 106(A7), 13,165, 2001.

Meredith, N. P., M. Cain, R. B. Horne, and D. Summers, Evidence for chorus-driven electron acceleration to relativistic energies from a survey of geomagnetically disturbed periods, J. Geophys. Res., 108(A6), 1248, doi:10.1029/2002JA009764, 2003.

Nagano, I., S. Yagitani, H. Kojima, and H. Matsumoto, Analysis of wave normal and Poynting vector of the chorus emissions observed by GEOTAIL, J. Geomag. Geoelectr., 48, 299, 1996.

Omura, Y. and D. Summers, Computer simulations of relativistic whistlermode wave-particle interactions, Phys. Plasmas, 11(7), 3530, 2004.

Santolik, O., D. A. Gurnett, J. S. Pickett, M. Parrot, and M. ComilleauWehrlin, Spatio-temporal structure of storm-time chorus, J. Geophys. Res., 108(A7), 1278, 2003.

Santolik, O., D. A. Gurnett, J. S. Pickett, M. Parrot, and M. ComilleauWehrlin, A microscopic and nanoscipic view of storm-time chorus on 31 March 2001, Geophys. Res. Lett., 31, L02801, doi:10.1029/ 2003GL018757, 2004.

Torrence, C. and G. P. Compo, A practical guide to wavelet analysis, Bull. Am. Met.Soc., 79(1), 61, 1998.

Tsurutani, B. T. and E. J. Smith, Postmidnight chorus: A substorm phenomenon, J. Geophys. Res., 79(1), 118, 1974.

Tsurutani, B. T. and E. J. Smith, Two types of magnetospheric ELF chorus and their substorm dependencies, J. Geophys. Res., 82, 5112, 1977.

Tsurutani, B. T. and G. Lakhina, Some basic concepts of wave-particle interactions in collisionless plasma, Rev. Geophys., 35(4), 491, 1997.

Tsyganenko, N., A model of the near magnetosphere with a dawn-dusk asymmetry. 1. Mathematical structure, J. Geophys. Res., 107(A8), 1179, doi:10.1029/2001JA000219, 2002.

Zhou, X.-Y., R. J. Strangeway, P. C. Anderson, D. G. Sibeck, B. T. Tsurutani, G. Haerendel, H. U. Frey, and J. K. Arballo, Shock aurora: FAST and DMSP observations, J. Geophys. Res., 108(A4), doi:10. 1029/2002JA009701, 2003.

O. P. Verkhoglyadova (e-mail: olgav@ucr.edu), B. T. Tsurutani, Y. Omura, and S. Yagitani 